\title{
Genetic and demographic parameters determining population persistence after a discrete change in the environment
}

\author{
ELIZABETH GRACE BOULDING* \& TOBY HAY \\ Department of Zoology, University of Guelph, Guelph, Ontario N1G 2W1 Canada
}

\begin{abstract}
Field studies suggest that populations often go extinct following discrete changes in the environment. However, populations may avoid extinction by rapidly adapting to their altered environment. We used a stochastic finite-locus model to estimate the distance the optimal value of a quantitative trait could shift in a single step $\Delta \theta_{\mathrm{c}}$ without causing more than $5 \%$ of the replicate populations to go extinct. We found that evolution increased the magnitude of $\Delta \theta_{\mathrm{c}}$ by at least two phenotypic standard deviations and that such evolution could take place within 5-10 generations. Indeed $\left(\Delta \theta_{\mathrm{c}}\right)^{2}$ increased approximately linearly with the logarithm of the initial population size and the rate of this increase was much greater when heritability was high or when stabilizing selection was weak. $\left(\Delta \theta_{\mathrm{c}}\right)^{2}$ also increased approximately linearly with the logarithm of per capita fecundity. To our surprise there was no 'demographic rescue' effect from migration; a population augmented with migrants from a neighbouring population where environmental conditions were unchanged was always more likely to go extinct. The addition of mutation, more loci, density-dependence, or environmental stochasticity had only small effects on the outcome. We were able to compare our results for closed populations with density-independent population growth to those from an analytical model and found good agreement so long as the proportion of the offspring surviving selection in the initial generations was at least $1 \%$.
\end{abstract}

Keywords: extinction risk, environmental stochasticity, fecundity, migration, rapid evolution, quantitative genetics.

\section{Introduction}

Most recent quantitative genetic models of wild populations have investigated how they might adapt or go extinct in response to continuous environmental change caused by global warming or similar gradual effects (Pease et al., 1989; Lynch et al., 1991; Charlesworth, 1993; Lynch \& Lande, 1993; Bürger \& Lynch, 1995; Kondrashov \& Yampolsky, 1996; Lande \& Shannon, 1996). However modern populations are also experiencing an accelerated rate of discrete environmental change caused, for example, by industrial disasters, habitat destruction, and the accidental introduction of exotic predators or competitors. Such abrupt change has been considered by Gomulkiewicz \& Holt (1995) who explicitly estimate the probability of extinction after a discrete shift in the optimal phenotype of a quantitative trait. They obtain an analytical solution by defining a critical population size above which the effect of

*Correspondence. E-mail: boulding@uoguelph.ca demographic stochasticity can be ignored, and then equate the risk of extinction with the amount of time a population spends below this critical population size. Gomulkiewicz \& Holt (1995) conclude that only large populations that experience relatively small environmental changes are likely to be rescued by evolution. We will show that their conclusion is too pessimistic and probably arose because they were unable to solve explicitly for the transient population dynamics.

The existing analytical models of adaptation to discrete changes in the environment (Lande, 1976; Gomulkiewicz \& Holt, 1995) do not include migration. This omission of migration is unrealistic, as we know that migration from a population where the optimal phenotype is different will constrain adaptation in quantitative genetic models of two populations of constant population size (Bulmer, 1980). On the other hand, models of population demography, in the absence of genetics, show that the presence of migration can sometimes prevent extinction (reviewed by Boyce, 1992). Demographic augmentation by migrants might be 
expected to be particularly important immediately after a change in the local environment if the focal population's instantaneous rate of population growth has temporarily become negative. Migrants might also provide an infusion of genetic variation that might increase the focal population's ability to respond to selection (Gomulkiewicz \& Holt, 1995).

The existing analytical models also do not directly include environmental and demographic stochasticity. This is a serious omission because these stochastic effects may cause the extinction of a population even if its mean intrinsic capacity for increase is positive (Lande, 1993). Further, Gomulkiewicz \& Holt's (1995) model does not include the effect of genetic drift. In addition, all previous models assume that population growth is density independent (Lande, 1976; Gomulkiewicz \& Holt, 1995).

We believe that a finite-locus model is particularly suitable for modelling short-term evolution of quantitative traits under strong natural selection because it models the loci themselves and therefore requires no assumptions about the distribution of the phenotypes after selection, the degree of selection-induced linkage disequilibrium, the shape of the fitness function, or constancy of additive genetic variance (e.g. Young, 1965; Boulding, 1990; Bürger \& Lynch, 1995). In addition, the use of a finite-locus model allows population size to vary, stochastic parameters to be easily incorporated, and the transient population dynamics to be obtained. An added benefit is that the probability of extinction for a given set of parameter values can be easily obtained by comparing replicate populations.

In this paper we first present a 'basic' stochastic finitelocus model and explore the effect of varying the magnitude of the shift in the optimum, the width of the fitness function, initial population size, heritability, and per capita fecundity. We then compare our basic finite-locus model to an analytical model from the literature. Finally, we extend our finite-locus model to cases that cannot be solved analytically, including the exchange of migrants with populations where the selective pressures are unchanged, logistic densitydependence, environmental stochasticity, changes in the number of loci determining the genetic value of the trait, and a nonzero mutation rate.

\section{Model}

We use a finite-locus model to model the evolution of a quantitative trait determined by $\mathrm{n}$ loci per individual in a finite population. Finite-locus models treat the genetic value for a quantitative trait for each individual as the sum of a series of loci with each locus having a defined effect (e.g. Uimari et al., 1996). We consider only a single trait under selection and assume that fitness can be described by a Gaussian function, that the trait measured in the environment before the shift in the optimum has the same genetic basis as the trait measured in the environment after the shift in the optimum (the genetic correlation is 1.0), that the population consists of outcrossing monoecious individuals such as an annual flower, and that a neighbouring population does not experience a change in its environment. An individual i from this population has a phenotype $z_{i}$ which is made up of a genetic component (or breeding value) $g_{i}$ and an environmental component $\mathrm{e}_{\mathrm{i}}$ :

$\mathrm{z}_{\mathrm{i}}=\mathrm{g}_{\mathrm{i}}+\mathrm{e}_{\mathrm{i}}$

In our basic model the genetic component was determined by 96 unlinked, diploid loci that combined additively; there was no dominance or epistasis. Each locus had one of the genotypes ' $A A$ ', ' $A a$ ', or ' $a a^{\prime}$ ' with each copy of ' $A$ ' contributing one to the breeding value of the organism and each ' $a$ ' contributing zero. The complete genotype of each member of the population was stored in computer memory as a string of bits. The environmental component was determined by a random normal deviate with a mean of zero and a variance equal to the desired environmental variance, $\sigma_{\mathrm{e}}^{2}$ (usually 4.5 model units).

The selection used in our model was hard or viability selection. The probability, $\mathrm{W}_{\mathrm{i}}$, of a juvenile surviving to become an adult was determined by the Gaussian fitness function:

$\mathrm{W}_{\mathrm{i}}=\exp \left(\frac{\left(\mathrm{z}_{\mathrm{i}} \theta\right)^{2}}{2 \sigma_{\omega}^{2}}\right)$

where $\theta$ is the optimal value of $\mathrm{z}$, and where $\sigma_{\omega}$ is the width (or standard deviation) of this Gaussian fitness function (see Lynch \& Lande, 1993). When $\bar{z}=\theta, \sigma_{\omega}{ }^{2}$ is inversely proportional to the strength of stabilizing selection towards the optimum.

This type of viability selection on the offspring incorporated demographic stochasticity into our model because in small populations it was possible for no offspring to survive even when $\overline{\mathrm{W}}$ was much larger than zero (see Gabriel et al., 1993). Another type of demographic stochasticity that arises from unequal sex ratios was not considered in our model, because the individuals were monoecious, but this could be important in species with separate sexes.

To initialize the population genotypic array, each allele of each individual in the population was assigned a value of $0(=A)$ or $1(=a)$ with a probability of $\mathrm{p}$ and $(1-p)$, respectively. In the basic model, we set $\mathrm{p}=0.5$ at each of 96 diploid loci $(n=96)$. This gave rise to a 
binomial distribution of genotypes with a mean of 96 'model' units, a range of 0-192 and an unscaled genetic variance of $2 n p(1-p)=48$. We realize that by setting $\mathrm{p}=0.5$ we maximize the initial additive genetic variance and make the population more able to evolve rapidly than if $\mathrm{p}$ were larger or smaller.

For ease of comparison to analytical models of continuous environmental change the initial mean phenotype was adjusted to 0.0 model units. However, model units are not easily compared with empirical results from wild populations or with analytical models, so many modellers report results in terms of phenotypic standard deviations (e.g. Bürger \& Lynch, 1995). We accomplished this by holding the initial phenotypic variance, $\sigma_{\mathrm{zi}}{ }^{2}$, constant at 9.0 model units, so that all other model parameters such as the shift in the optimum and the intensity of selection could be reported in units of phenotypic standard deviations. To hold the phenotypic variance constant at 9.0 model units in the first generation we scaled the initial additive genetic variance. For example, when the initial heritability was 0.5 we scaled the initial raw genetic variance of 48 model units to an initial value of 4.5 model units. For the basic model this was done in the same way for all generations of all runs; the breeding value of each offspring was adjusted by multiplying the unscaled adjusted breeding value by $(4.5 / 48)^{1 / 2}$ just before selection took place. We then adjusted the environmental variance to a value of 4.5 model units. These adjustments did not affect the results and allowed comparison of models with different numbers of loci.

For all simulations we created an initial distribution at generation 0 that was approximately Gaussian with a mean (after scaling) of 0 model units. We did this by carrying out one generation of weak selection with the heritability set to 1.0 and the fitness function having a mean at the initial optimum of 0 model units, and a variance, $\sigma_{\omega}{ }^{2}=5.0$ model units. To test whether populations better adapted to the initial optimum had a different risk of extinction we experimented with holding the optimum at zero for 20 generations. However, we found no difference in the probability that the populations would adapt to the new optimum.

Following this initial generation, the optimum of the fitness function was shifted to its new value in a single step. The default value for the distance the optimum was shifted, $\Delta \theta$, was 9 model units or 3 phenotypic standard deviations $\left(\sigma_{\mathrm{zi}}\right)$. In the basic model the fitness function had a default variance of $\sigma_{\omega}^{2}=5.0$ model units, and therefore a standard deviation, $\sigma_{\omega}$, of $0.75 \sigma_{\mathrm{zi}}$, which we calculated to be equivalent to an intensity of stabilizing selection of $\mathrm{j}=-0.4$ (Endler, 1986). The available empirical data suggest that is not unrealistically strong stabilizing selection. Endler (1986) reviewed 165 examples of stabilizing selection on quantitative traits of wild populations and found a range of $j=-0.1$ to -0.9 when $\mathrm{j}$ was calculated as $\mathrm{j}=\left(\mathrm{v}_{\mathrm{a}}-\mathrm{v}_{\mathrm{b}}\right) / \mathrm{v}_{\mathrm{b}}$, where $\mathrm{v}_{\mathrm{b}}$ is the variance before selection and $\mathrm{v}_{\mathrm{a}}$ is the variance after selection. More than $18 \%$ of his empirical examples were under equally strong stabilizing selection as that used in standard parameter set in our model $(\mathrm{j}<-0.4)$. However, we also experimented with using a wider fitness function so that selection was weaker. In different runs of our basic model we varied $\sigma_{\omega}^{2}$ from 0.28 to $3.2 \sigma_{\mathrm{zi}}$.

As is appropriate for a quantitative trait, both the initial and the new optimum in our model were far from the extremes of the distribution of possible breeding values. This ensured that changes in the phenotypic mean were decoupled from those in the phenotypic variance, something that has been predicted theoretically (Slatkin, 1978) and that we also observed empirically in our finite-locus model.

The initial population size at generation 0 was always at the carrying capacity, $\mathrm{N}_{\max }$, which had a default value of 1000 individuals, which each had a default value for fecundity, $\mathrm{f}$, of 3 . In succeeding generations population size was allowed to vary but was not permitted to exceed the carrying capacity. The maximum number of offspring that could be produced was the minimum of $\mathrm{N}_{\max }$ and $\mathrm{N} \times \mathrm{f}$, where $\mathrm{N}$ was the current population size of the parent generation.

During 100 generations of strong selection the amount of additive genetic variation $\sigma_{\mathrm{g}}{ }^{2}$ in our populations declined slightly, but this had little effect on the important events in the first 10 generations. For that reason we kept the mutation rate, $\mu$, at zero for the basic model. However, we explored the effects of varying the mutation rate in extensions of the model.

\section{Simulation of a generation}

In the basic model the ordering of events in each generation was: (1) reproduction and viability selection; (2) death of the parental generation; and (3) a census of the population size. Generations were assumed to be nonoverlapping. During each generation offspring were produced by randomly choosing two monoecious parents and allowing their gametes to fuse. There was no linkage among loci; the gamete created by each parent contained one allele chosen at random from the two at each genetic locus. The $z_{i}$ of each offspring was then calculated and used to calculate $\mathrm{W}_{\mathrm{i}}$. The offspring survived if $\mathrm{W}_{\mathrm{i}}>\mathrm{v}$, where $\mathrm{v}$ is a uniform random number between 0 and 1 . Regardless of their offspring's fate, we continued to sample parental couples with replacement, who were allowed to produce one offspring that either survived or failed to survive, either until the number of surviving offspring equalled $\mathrm{N}_{\max }$ or until the total number of matings reached $f \times N$. The individuals making up the 
parent generation then died and their offspring became the current generation. The population was then censused. If the new population size was two or less the population was considered to have gone extinct and the simulation was stopped. In the extension of the basic model that included migration, migration occurred right after the census. The complete cycle was repeated for each generation until the population had gone extinct or until the population had recovered to the carrying capacity.

We usually ran 500 or more replicate runs for each combination of parameter values in the basic model. We then used database programs to calculate the probability of extinction, the mean population size (and standard error) and the mean phenotype (and standard error) for a given generation. The model was written in $\mathrm{C}$ and run on the University of Guelph's Silicon Graphics Challenge-XL computer. The $\mathrm{C}$ source code is available from the authors.

In the basic model we used a range of values for the parameters $\sigma_{\omega}$ (from 0.28 to $3.2 \sigma_{\mathrm{zi}}$ ), f (from 2 to 10000 ), $\mathrm{h}^{2}$ (from 0.001 to 1.0 ) and $\mathrm{N}_{\mathrm{i}}$ (from 10 to 100 000). For each combination of these parameters we varied the shift in the optimum $\Delta \theta_{\mathrm{c}}$, to determine what shift would lead to $95 \%$ of the simulated populations surviving. This required thousands of simulations at various combinations of parameter values as $\Delta \theta_{\mathrm{c}}$ was shifted nearer and nearer to the critical value.

\section{Extensions of the basic model}

Migration We incorporated migration from a neighbouring population where the optimum was unchanged in a two-island extension of our finite-locus model. When the migration rate was nonzero, migration occurred right after the population census, with $\mathrm{m} \times \mathrm{N}_{1}$ individuals leaving the focal population for the neighbouring population and $\mathrm{m} \times \mathrm{N}_{2}$ individuals leaving the neighbouring population for the focal population.

Density-dependence We incorporated density-dependence using the equation:

$\mathrm{f}\left(\mathrm{N}_{\mathrm{t}}\right)=\left(\begin{array}{lll}1.5 & \mathrm{~b}\left(\mathrm{~N}_{\mathrm{t}}\right. & \mathrm{N}_{\max }\end{array}\right)$

where $f\left(N_{t}\right)$ measures fecundity, which changes with $N$, and $b$ is a constant that measures how fast $f\left(N_{t}\right)$ decreases with increasing population size. $f\left(N_{t}\right)$ in this extension of our model is equivalent to $R_{o}$ in the usual analytical difference equation for population growth with a discrete, density-independent model (i.e. $\mathrm{N}_{\mathrm{t}+1}=$ $\mathrm{R}_{\mathrm{o}} \mathrm{N}_{\mathrm{t}}$ ). We present the data for the case for less fecund organisms when $b=0.0015$, which is approximately equivalent to a per capita fecundity of 3.0 when population densities are very low, and of 1.5 when population densities are close to $\mathrm{N}_{\max }$. As before, only one offspring is produced per mating event and mating stops once the number of matings reaches $\mathrm{N}_{\max } \times f\left(\mathrm{~N}_{t}\right)$.

Environmental stochasticity We incorporated 'year to year' environmental stochasticity in birth-rates into the finite-locus model by sampling fecundity so that the mean fecundity was still 3.0, but so that fecundity had an equal probability of taking one of two discrete values in each generation. When the environmental stochasticity was 'moderate' that meant that during a particular generation there was a probability of $50 \%$ that $f=2$ and a probability of $50 \%$ that $\mathrm{f}=4$, which results in a variance in fecundity of 1.0 . When the environmental stochasticity was 'high', there was a probability of $50 \%$ that $\mathrm{f}=1$ and a probability of $50 \%$ that $\mathrm{f}=5$, resulting in a variance of 4.0 .

Mutation We incorporated mutation into an extension of our model where it was assumed to occur at a constant rate per genome of $\mu$, which we varied from 0.01 to 0.1 . For example $\mu=0.1$ was implemented by choosing one out of 10 individuals at random, choosing one allele at one of their loci at random, and, depending on its initial state, either changing ' $A$ ' to ' $a$ ' or ' $a$ ' to ' $A$ '. Therefore, for the basic model with its 96 diploid loci, $\mu=0.1$ would give a genic mutation rate of $5.2 \times 10^{-4}$. It is important to note that the population was unlikely to be in mutation/selection/drift/migration balance even in extensions of our basic model that had mutation. This was deliberate as we wanted to manipulate heritability and selection independently of population size. Indeed wild populations are probably often not in mutation-selection-drift-migration equilibrium because of past population bottlenecks (Lynch, 1996).

Mutation-selection balance We experimented with beginning the simulations with the populations in mutation-selection-drift balance. We accomplished this by fixing the selection intensity and then determining, by trial and error, the mutation rate that would keep the population in balance. We concluded that a population was in mutation-selection-drift balance if the additive genetic variance stayed within $5 \%$ of a constant value for 1000 generations when the fitness function was held at the original optimum. Once we had determined that the appropriate value for $\sigma_{\omega}=0.75 \sigma_{\mathrm{zi}}$ with the usual standard parameter values was $\mu=0.5$, we ran 10000 simulations but held $\theta=0.0$ for 20 generations before shifting it to $3.0 \sigma_{\mathrm{zi}}$. Similarly we ran 10000 simulations with $\sigma_{\omega}=3.2 \sigma_{\mathrm{zi}}$ and $\mu=0.1$, but held $\theta=0.0$ for 20 generations before shifting it to $6.37 \sigma_{\mathrm{zi}}$. We then compared the percentage of replicate simulations that persisted for 100 generations. 
Number of loci To determine how sensitive our results were to details of the genetics, we also ran a series of simulations that varied the number of loci determining the breeding value of the trait from 8 to 1024 , while holding the initial value of $\sigma_{\mathrm{gi}}{ }^{2}=4.5$ model units.

\section{Analytical model}

To obtain the analytical approximation of our basic finite-locus model we used eqn 14 in Lande (1976) modified so that the new optimum was not necessarily at zero to get an expression for the mean phenotype, $\bar{z}$ :

$\overline{\mathrm{z}}_{\mathrm{t}+1}=\frac{1 \mathrm{~h}^{2} \sigma_{z}^{2}}{\sigma_{\omega}^{2}+\sigma_{\mathrm{z}}^{2}\left(\overline{\mathrm{z}}_{\mathrm{t}} \quad \theta\right)+\theta}$

We then slightly modified equations from Lande (1976), Latter (1970), Charlesworth (1993), and Gomulkiewicz $\&$ Holt (1995) so that the maximum fitness was equal to the fecundity to get an expression for the mean fitness at time $\mathrm{t}, \overline{\mathrm{W}}_{\mathrm{t}}$ :

$\overline{\mathrm{W}}_{\mathrm{t}}=\mathrm{W}_{\max }\left(\frac{\sigma_{\omega}^{2}}{\sigma_{\omega}^{2}+\sigma_{\mathrm{z}}^{2}}\right)^{\frac{1}{2}} \exp \left(\frac{\left(\overline{\mathrm{z}}_{\mathrm{t}} \theta\right)^{2}}{2\left(\sigma_{\omega}^{2}+\sigma_{\mathrm{z}}^{2}\right)}\right)$

We then substituted eqn 5 into the expression for a density-independent population with discrete generations (Lande, 1976):

$\mathrm{N}_{\mathrm{t}+1}=\overline{\mathrm{W}}_{\mathrm{t}} \mathrm{N}_{\mathrm{t}}$.

where $\mathrm{N}_{\mathrm{t}}$ is the population size at time $\mathrm{t}$.

This analytical approximation gave us deterministic results for the mean phenotype, $z$, and the population size, $N$, for a particular time $t$. This allowed us to plot the trajectories of population sizes predicted by this analytical approximation on the same graph as the mean population sizes observed in our basic finite-locus model. We have not derived and are not aware of analytical approximations that could be compared with extensions of our finite-locus model that directly incorporate migration, environmental stochasticity or density-dependence.

\section{Results}

\section{General behaviour of finite-locus model under selection}

For the default parameter values used in our basic model, the fate of a population was usually decided during the first few generations after the change in the environment. If the population was capable of evolving to a new optimum it happened very quickly. The initial drop in population size increased with the magnitude of the shift in the optimal phenotype, as did the time it took for the population size to recover to carrying capacity. The fate of the populations was deterministic for most of parameter space. For the shifts of one phenotypic standard deviation or less, the population size did not drop below the carrying capacity and there was no risk of extinction. Indeed no extinctions were observed until the shift in the optimum was greater than three phenotypic standard deviations and the initial drop in population size was very large. None of the replicate 500 populations managed to shift five phenotypic standard deviations. The largest shift in the optimum that $95 \%$ of the populations could adapt to was about 3.05 phenotypic standard deviations.

\section{Effect of population parameters}

The initial drop in population size one generation after the shift in the optimum was always by the same proportion of the population's initial size and was almost identical for different initial heritability values (Fig. 1). For the default parameter values used in the basic model the initial drop was to about $8 \%$ of the initial population size (Fig. 1). Populations of 50 dropped to a mean minimum population size of 4 and consequently had a higher probability of extinction from demographic stochasticity $\left(84 \%\right.$ for $\mathrm{h}^{2}=0.5$ and $35 \%$ for $\mathrm{h}^{2}=1.0$ ) than populations of 500 which dropped to a mean minimum population size of $40\left(11 \%\right.$ for $\mathrm{h}^{2}=0.5$ and $0 \%$ for $h^{2}=1.0$ ) or populations of 5000 which dropped to a mean minimum population size of $400(0 \%$ for $h^{2}=0.5$ and $0 \%$ for $h^{2}=1.0$ ). However, the heritability substantially affected the probability of extinction. Populations with lower initial heritabilities took longer to adapt to the new optimum and consequently spent more generations at a small population size than did populations with high levels of genetic variation (Fig. 1). This increased the risk of extinction for populations of 50 or 500 individuals (Fig. 1a,b).

Note that the populations that go extinct do so because too few offspring are surviving in the new environment and not because there is too little additive genetic variance to respond to selection. Indeed in this example the additive genetic variance is still at $90 \%$ of its initial value just before a population goes extinct. When we followed allele frequencies at individual loci we found that no loci became fixed unless the mean population size dropped to very low levels (10 or fewer individuals) and stayed there for several generations. However this fixation was entirely attributable to genetic drift as it happened at the same rate for loci under selection and those not under selection. We could detect this because we also monitored some loci that were not 
(a)

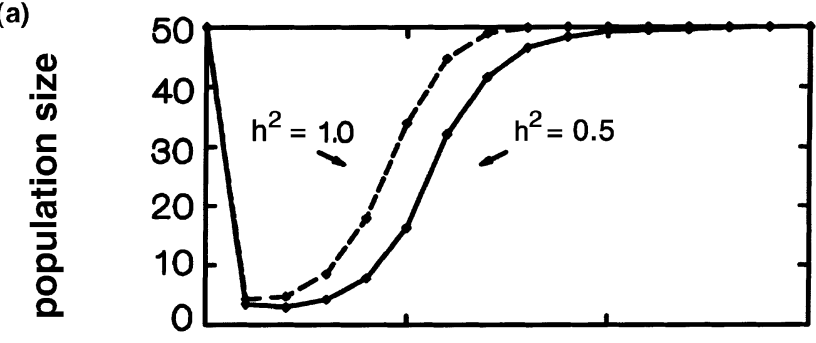

(b)

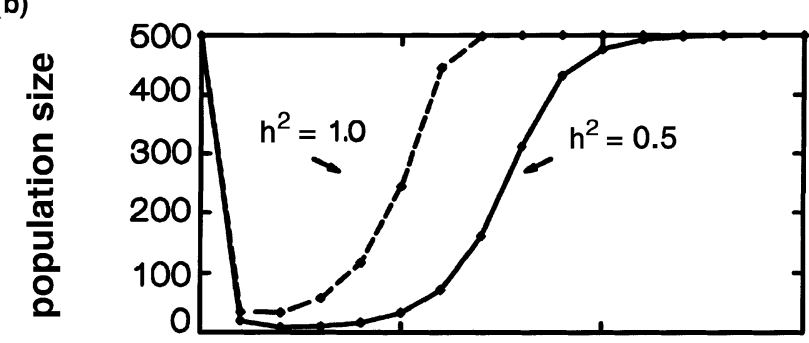

(c)

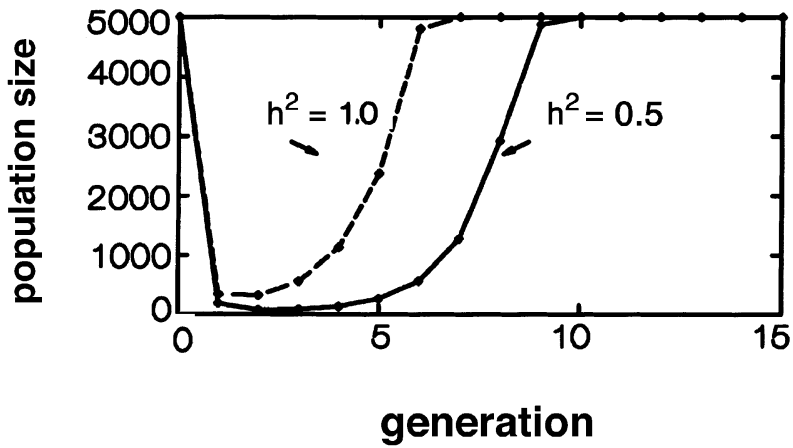

Fig. 1 The effect of heritability on the transient population dynamics after a discrete change in the environment. The mean population size for each generation is plotted for all populations that survived by adapting to the change in the environment. The percentage of populations surviving was $\mathrm{N}_{\mathrm{i}}=50$ : $16 \%$ for $\mathrm{h}^{2}=0.5,65 \%$ for $\mathrm{h}^{2}=1.0 ; \mathrm{N}_{\mathrm{i}}=500: 89 \%$ for $\mathrm{h}^{2}=0.5$, $100 \%$ for $h^{2}=1.0 ; \mathrm{N}_{\mathrm{i}}=5000: 100 \%$ for $\mathrm{h}^{2}=0.5,100 \%$ for $\mathrm{h}^{2}=1.0$. Other parameter values as in basic model.

under selection because they were among the 928 out of 1024 loci not used in the basic model.

The square of the shift in the optimum to which $95 \%$ of the populations could adapt to $\left(\Delta \theta_{c}\right)^{2}$, increased approximately linearly with the logarithm of initial population size (Fig. 2). The rate of increase in $\Delta \theta_{\mathrm{c}}$ with population size was significantly greater when the initial heritability was high (Fig. 2a). In the results given below we refer to the difference between the value of $\Delta \theta_{\mathrm{c}}$ for a very low heritability and the value for a high heritability as a measure of how much difference evolution made to the amount of environmental change the populations could withstand. When the initial population size was 10000 , increasing the heritability from 0.001 to 1.0 increased $\Delta \theta_{\mathrm{c}}$ from $1.4 \sigma_{\mathrm{zi}}$ to $4.2 \sigma_{\mathrm{zi}}$, an increase of
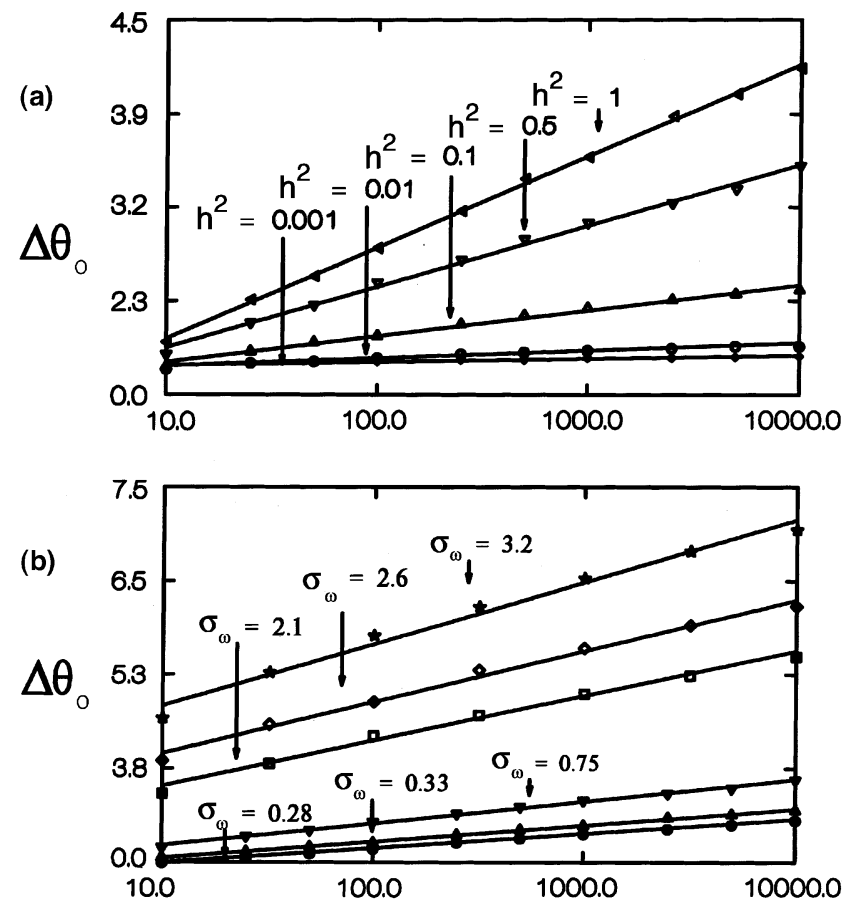

Initial population size

Fig. 2 The importance of initial population size, heritability, and width of the fitness function in increasing the magnitude of the shift in the optimum that $95 \%$ of the populations were able to withstand, $\Delta \theta_{\mathrm{c}}$. Other parameter values as in basic model. To make the plots linear the $x$-axis has a logarithmic base 10 scale and the $y$-axis has an exponent of 2 scale. a. The importance of evolution: $\theta_{\mathrm{c}}$ in phenotypic standard deviations, $\sigma_{\mathrm{zi}}$, plotted against initial population size for different heritability values. The slopes of the lines which are $0.174,0.403$, $1.38,3.29,4.94$ for the heritabilities of $0.001,0.01,0.1,0.5,1.0$, respectively, are significantly heterogeneous, $P<0.001$.

b. The effect of the strength of stabilizing selection: $\Delta \theta_{\mathrm{c}}$ in $\sigma_{\mathrm{zi}}$ plotted against initial population size for different widths of the fitness function (in $\sigma_{\mathrm{zi}}$ ). The slopes of the lines which are $2.15,2.43,3.29,6.73,7.71,9.33$ for the fitness functions with standard deviations of $0.28,0.33,0.75,2.1,2.6,3.2$, respectively, are significantly heterogeneous, $P<0.001$.

nearly three phenotypic standard deviations (Fig. 2a). Fecundity was even more important. At fecundities of $10000, \Delta \theta_{\mathrm{c}}$ was increased by evolution by as much as six phenotypic standard deviations (Boulding \& Hay, unpublished data).

The rate of increase in $\Delta \theta_{c}$ with initial population size was significantly greater when the fitness function was wider, so that stabilizing selection was less intense, than when it was narrow (Fig. 2b). When the width of the fitness function was $3.2 \sigma_{\mathrm{zi}}$, increasing the initial population size from 10 to 10000 increased $\Delta \theta_{\mathrm{c}}$ by $3.55 \sigma_{\mathrm{zi}}$ (Fig. 2b) whereas when the default width of the fitness function was used $\left(0.75 \sigma_{\mathrm{zi}}\right)$, it increased $\Delta \theta_{\mathrm{c}}$ by $3.15 \sigma_{\mathrm{zi}}$ 
(Fig. 2b). When the fitness function was wide, the populations could tolerate a much larger shift in the optimum without going extinct (Fig. 2b) but the populations took much longer to adapt to the larger shifts ( $>100$ generations for $\Delta \theta_{\mathrm{c}}=6.6 \sigma_{\mathrm{zi}}$ and $\sigma_{\omega}=3.2 \sigma_{\mathrm{zi}}$ ).

A high rate of evolution, made possible by a high heritability, was particularly important in preventing extinction when the initial population size was moderately large (Fig. 2a). Increasing the heritability from 0.001 to 1.0 when the initial population size was only 10 increased $\Delta \theta_{\text {c }}$ by $0.234 \sigma_{\text {zi }}$ (Fig. 2a). However the same increase in heritability at the default initial population size $(N=1000)$ increased $\Delta \theta_{\mathrm{c}}$ by $2.16 \sigma_{\mathrm{zi}}$ (Fig. 2a) and at $N=10000$ increased $\Delta \theta_{\text {c }}$ by $2.75 \sigma_{\text {zi }}$ (Fig. 2a). A high heritability was even more effective at preventing extinction when the fitness function was wider than the default value of $0.75 \sigma_{\mathrm{zi}}$. When the width of the fitness function was $3.2 \sigma_{\mathrm{zi}}$ and the initial population size was the default value $(N=1000)$, increasing the heritability from 0.001 to 1.0 increased $\Delta \theta_{\mathrm{c}}$ by $2.36 \sigma_{\mathrm{zi}}$ (Boulding and Hay, unpublished data).

Gomulkiewicz \& Holt (1995) define a measure of initial maladaptation after the optimum has shifted, $\beta_{0}=(\Delta \theta)^{2} /\left(\sigma_{\mathrm{z}}^{2}+\sigma_{\omega}^{2}\right)$, where $\Delta \theta$ is the amount the optimum has shifted, $\sigma_{\mathrm{z}}{ }^{2}$ is the phenotypic variance and $\sigma_{\omega}{ }^{2}$ is the variance of a Gaussian fitness function. However we decided to hold $\sigma_{\mathrm{zi}}$ constant and use $\left(\Delta \theta_{\mathrm{c}}\right)^{2}$ as a measure of the difficulty of shifting to a new optimum rather than their $\beta_{0}$ because we found that $\beta_{0}$ did not adequately correct for differences in $\sigma_{\omega}$. When $\beta_{0}$ was calculated from the values of $\Delta \theta_{\mathrm{c}}$ shown in Fig. $2 \mathrm{~b}$ and plotted against the logarithm of initial population size, those runs with wide fitness functions had significantly shallower slopes than those with narrower fitness functions (Boulding and Hay, unpublished data).

For the default shift in the optimum used in the basic model (i.e. $\Delta \theta=3 \sigma_{\mathrm{zi}}$ ), $100 \%$ of the populations adapted successfully when the width of the fitness function, $\sigma_{\omega}$, was greater than $0.24 \sigma_{\mathrm{zi}}$. Below this width of the fitness function, populations had a decreasing probability of adapting, which reached zero when the width became narrower than $0.17 \sigma_{\mathrm{zi}}$. A very narrow fitness function $\left(\sigma_{\omega}=0.22 \sigma_{\mathrm{zi}}\right)$ delayed the return of the population to carrying capacity until long after the population mean had reached the optimum; the mean phenotype reached the new optimum, two phenotypic standard deviations away, in less than four generations but it then took 50 generations of intense stabilizing selection to erode the additive genetic variance sufficiently to permit the population size to increase all the way back up to the carrying capacity.

\section{Comparison with analytical model}

There was a close correspondence between the transient population dynamics of the finite-locus model and those predicted by the analytical model when selection was intense $(25 / 3000$ offspring $(0.8 \%)$ surviving viability selection in generation 1 (Fig. 3a,b)) but not when viability selection was extremely intense $(340 /$ 10000000 offspring $(0.003 \%)$ surviving viability selection in generation 1 (Fig. 3c,d)). When selection was extremely intense the mean phenotype did not approach

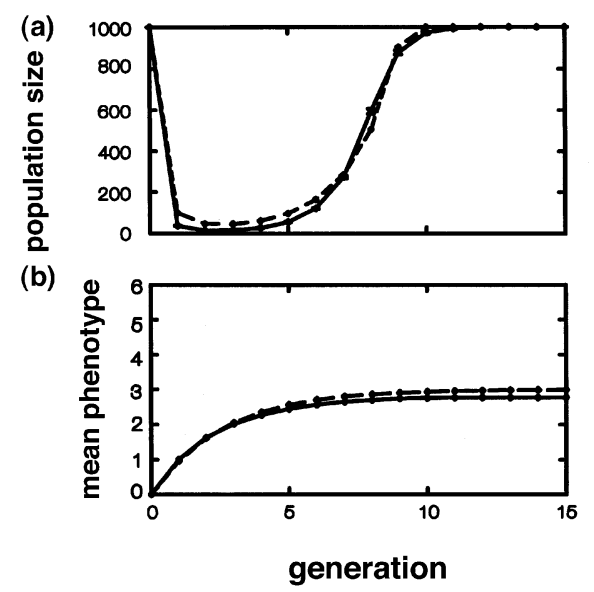

(c)

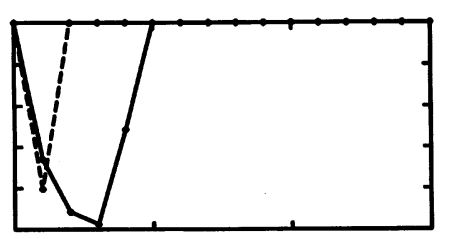

(d)

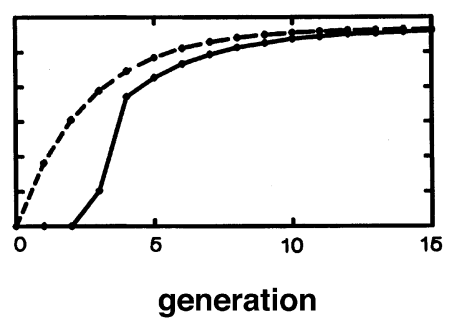

Fig. 3 Comparison of the mean of 500 replicates of our finite locus simulation model (solid line) with a deterministic analytical model (dotted line, see text). Other parameter values as in basic model. a. The population size when the fecundity, f, and shift in the optimum, $\Delta \theta$, is as in the basic model (i.e. $\mathrm{f}=3$ and $\Delta \theta=3.0 \sigma_{\mathrm{zi}}$ ). b. The phenotype when the fecundity and shift in the optimum is as in the basic model (i.e. $\mathrm{f}=3$ and $\Delta \theta=3.0 \sigma_{\mathrm{zi}}$ ). c. The population size when the fecundity is very high and shift in the optimum very large so that viability selection is extremely intense $\left(\mathrm{f}=10000\right.$ and $\left.\Delta \theta=5.66 \sigma_{\mathrm{zi}}\right)$. $\mathrm{d}$. The phenotype when the fecundity is very high and shift in the optimum very large so that viability selection is extremely intense $\left(\mathrm{f}=10000\right.$ and $\left.\Delta \theta=5.66 \sigma_{\mathrm{zi}}\right)$.

(C) The Genetics Society of Great Britain, Heredity, 86, 313-324. 
the new optimum geometrically. Instead it stayed close to the original optimum at zero until by chance some extreme offspring were produced. The transition to the new optimum then usually followed quickly within a couple of generations especially if the fecundity was high.

The populations in the finite-locus model consistently dropped to a slightly smaller size than that predicted by the analytical model (Fig. 3a,c). This probably occurred because the mean phenotype approaches the optimum at a slightly lower rate in the finite-locus model than in the analytical model (Fig. 3b,d). Indeed additional comparisons show that the predictions of $\Delta \theta_{\mathrm{c}}$ from the analytical model are always more optimistic than those of the finite-locus model and that this discrepancy increases at high fecundities where selection is more intense. For both the finite-locus model and the analytical model $\left(\Delta \theta_{\mathrm{c}}\right)^{2}$ increased approximately linearly with the logarithm of the fecundity but the rate of increase was much less for the finite-locus model. For a high fecundity of 3162 (and the other parameters as in the basic model) our finite-locus model gives $\left(\Delta \theta_{\mathrm{c}}\right)^{2}=26.7 \sigma_{\mathrm{zi}}$ but the analytical model gives $\left(\Delta \theta_{\mathrm{c}}\right)^{2}=42.8 \sigma_{\mathrm{zi}}$.

\section{Extensions of the basic model}

Migration Migrants from a population where the optimum was unchanged never helped the focal population adapt to the new optimum. Instead the percentage of populations that survived decreased from $100 \%$ to $0 \%$ as the two-way migration rate increased from 0.01 to 0.1 (Fig. 4 b). As the migration rate increased from 0.01 to 0.1 the initial drop in population size, which was the most pronounced at generation two, increased (Fig. 4b). This occurred because the population lost individuals that were partially adapted to local conditions as emigrants and gained individuals that were not adapted as immigrants. Indeed the mean population size drops to zero when the migration rate is from 0.08 to 0.1 because the focal population has gone extinct (Fig. 4a). This local extinction of the focal population occurs because the census is taken before the immigrants from the unshifted population arrive. An interesting but trivial increase in the percentage of populations surviving occurs when the migration rate exceeds 0.1 (Fig. 4a). In that case there were so many immigrants coming into the focal population from the unshifted population that a proportion of their offspring survived to be censused the following generation (Fig. 4b). This is a trivial result because the focal population has been converted into a 'sink' population that will not persist if immigration is stopped. We observed very similar results with another version of the model that had only one-
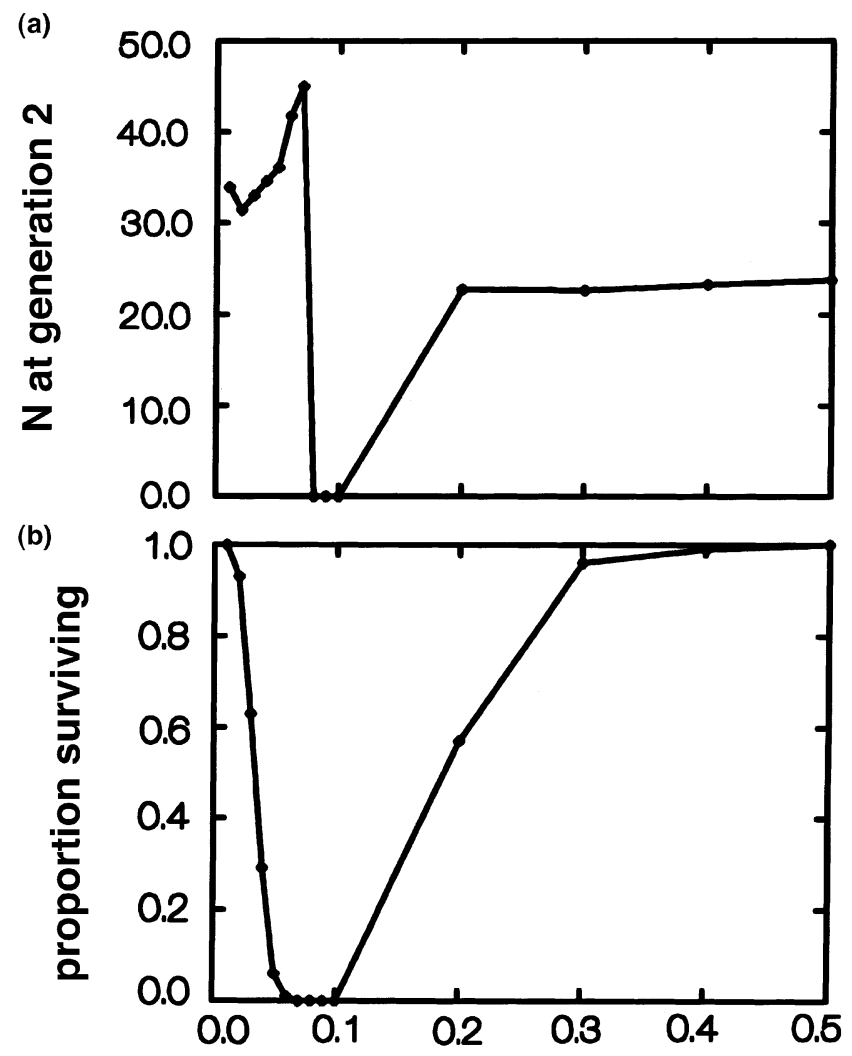

migration rate

Fig. 4 The effect of the migration rate, m, from another population where the optimal phenotype is unchanged on a population's potential to adapt to a new optimal phenotype. Both the shifted and the unshifted population exported $\mathrm{m} \times \mathrm{N}$ migrants to the other population (see text). For these populations, the optimum was shifted 2.0 phenotypic standard deviations, $\sigma_{\mathrm{zi}}$, and the width of the fitness function was $0.33 \sigma_{\mathrm{zi}}$. Other parameter values as in the basic model. a. The population size two generations after the shift in the optimum. b. The proportion of the 500 populations surviving at generation 100 .

way migration from the neighbouring population to the focal population.

Effect of density-dependence The incorporation of density-dependent fecundity instead of density-independent fecundity made only a small difference to the dynamics of the model. This occurred because it is the reproductive rate at low population densities that is important in determining whether populations will adapt. The density-dependent population recovered almost as quickly from the initial drop as did the population with a density-independent fecundity of two. The densitydependent populations were much less likely to go extinct (survival $=83 \%$ ) than density-independent 
populations with a fecundity of one (survival $=0 \%$ ) but were slightly more likely to go extinct than densityindependent populations with a fecundity of two (survival $=100 \%$ ).

Environmental stochasticity Incorporation of environmental stochasticity into the model always increased the risk of extinction. When the environmental stochasticity was high (the mean per capita fecundity was set to one in a bad year and five in a good year) the probability of survival of 1000 replicate simulations was only $58.0 \%$. When the environmental stochasticity was moderate (the mean per capita fecundity was set at two in a bad year and four in a good year) the probability of survival increased significantly to $90.2 \%$. Those simulations without environmental stochasticity had an even higher probability of survival of $96.5 \%$.

When we shifted the optimum even further $\left(\Delta \theta_{\mathrm{c}}=3.46 \sigma_{\mathrm{zi}}\right)$ we found a similar pattern. The simulations with high environmental stochasticity had a probability of survival of only $12.7 \%$, those with moderate environmental stochasticity had a significantly higher probability of survival of $20.1 \%$, and those without environmental stochasticity had the highest probability of survival of $23.4 \%$.

Mutation The mutation rate seemed to have little effect on the short-term behaviour of the model. An increase in the per genome mutation rate in the basic model from 0 to 0.5 made less than a $0.5 \%$ difference in the proportion of the replicate simulations that reached the new optimum. This would be expected when the number of loci is moderately large since the change in additive genetic variance will be dominated by a shift in linkage disequilibria rather than changes in allele frequencies when the number of loci is large (Bulmer, 1980).

Mutation-selection balance The simulations that were initially in mutation-selection-drift equilibrium were not significantly more likely to go extinct than matching simulations with a mutation rate of zero. When $\sigma_{\omega}=0.75 \sigma_{\mathrm{zi}}$ the proportion of the populations surviving to 100 generations was $94.3 \%$ when $\mu=0.5$ and $93.6 \%$ when $\mu=0.0$. When $\sigma_{\omega}=3.2 \sigma_{\mathrm{zi}}$ the proportion of the populations surviving to 100 generation was $94.3 \%$ when $\mu=0.1$ and $94.0 \%$ when $\mu=0.0$.

Number of loci The details of the underlying genetics of the finite-locus model seemed to have little effect on the short-term results. The number of loci had very little effect on the population size 10 generations after a shift in the optimum. The number of loci also had very little effect on the rate at which the mean population phenotype approached the optimum.

\section{Discussion}

Our finite-locus model showed that large, fecund populations with high levels of genetic variation could withstand larger discrete changes in the optimal value of a quantitative trait without going extinct than could smaller, less fecund populations with low levels of genetic variation. Previous authors have pointed out that the demographic costs imposed on a population will increase as the population mean is shifted further from the optimum and that there will be a limit to what a population with particular parameters can tolerate (Charlesworth, 1993; Bürger \& Lynch, 1995).

Highly fecund populations were more likely to produce individuals with rare extreme genotypes close to the new optimum. Indeed both our finite-locus model and the analytical model showed that the square of the shift in the optimum that a population could adapt to increased approximately linearly with the logarithm of fecundity. However, rapid evolution of 10 or more phenotypic standard deviations has also been observed for less fecund organisms in laboratory experiments as a result of artificial truncation selection (Falconer \& Mackay, 1996). This suggests that if the optimum phenotype for a wild population shifted five phenotypic standard deviations in a series of small discrete steps rather than in a single step that the population would be less likely to go extinct, especially if there was enough time in between successive steps for the population to return to carrying capacity. Thus wild populations might manage to survive large discrete changes of 10 or more phenotypic standard deviations in a series of steps.

We also found that the square of the shift in the optimum that $95 \%$ of the populations could tolerate increased approximately linearly with the logarithm of initial population size and the rate of this increase was greater at high heritabilities. This is not unexpected as the initial phenotypic distribution just before a shift in the optimum is approximately Gaussian so that the number of individuals at a distance, $\Delta \theta$, from the optimum might be expected to be a function of $\mathrm{N}_{\mathrm{i}} \times \mathrm{e}^{-(\Delta \theta) 2}$. As predicted by our analytical approximation, the population size in the first generation was reduced by a constant proportion for a given shift in the optimum regardless of the heritability. Nevertheless, after the first generation heritability made a difference and populations with high initial heritabilities could tolerate larger shifts in the optimum because their rate of adaptation was greater. A greater rate of adaptation meant that the population growth rate became positive sooner and the population spent fewer generations at small population sizes where it was vulnerable to extinction from demographic stochasticity. Thus we do not agree with 
Lande's (1988) conclusion that demography is more important than additive genetic variance over the short term; we would argue that, given the current high probability of discrete environmental change, both are important. An exception to higher levels of additive genetic variation increasing fitness occurred when the fitness function was very narrow, as has previously been shown by an analytical model (Lande \& Shannon, 1996).

We had expected that low rates of migration from neighbouring populations where the environment had not changed would decrease the probability of extinction because we had believed that this constraint on adaptation of the focal population would be offset by the demographic augmentation at small population sizes. Instead we found that the percentage of focal populations that survived decreased from $100 \%$ to $0 \%$ as the migration rate increased from 0.01 to 0.1 and that populations fared best with no migrants at all. A somewhat similar case was investigated analytically by Kirkpatrick \& Barton (1997), who found that populations on the periphery of a species' range were constrained from adapting to local conditions and became demographic sinks.

Before we can fully evaluate how important evolution is in preventing extinction in wild populations, we need to know how common step-like shifts of 1-4 phenotypic standard deviations are in nature. Unfortunately there have been few field studies where both the demography and genetics of a population have been followed after a change in the environment has resulted in a discrete shift in the optimal phenotype. One exception to this is the long-term studies of Darwin's finches by P. R. Grant and colleagues on the island of Daphne Major in the Galapagos. During a temporary drought in 1977 the optimal beak depth for one species of Galapagos finch, Geospiza fortis, shifted from approximately $9.4 \mathrm{~mm}$ to $13 \mathrm{~mm}$ (Grant, 1986, p. 213) - about four phenotypic standard deviations - because the only seeds available as food were large with hard shells (Boag \& Grant, 1981). The population size of the finches dropped from 642 to 85 birds and the mean bill depth of the surviving adults increased to $9.96 \mathrm{~mm}$ from its value of $9.42 \mathrm{~mm}$ before the drought (Boag \& Grant, 1981). It seems likely that if the drought had continued the population might have gone extinct. Their work shows that discrete shifts of four phenotypic standard deviations in the optimal phenotype do occur in nature and that they can result in drops in the population size.

Populations that undergo abrupt discrete shifts have received little recent attention from theoreticians because the genetics seems simple. However, our finite-locus model has shown that the genetics is not simple if selection is extremely intense or if migration is involved. When the shift in the optimum was so large that less than $1 \%$ of the juveniles survived, the mean phenotype no longer approached the new optimum geometrically and the analytical approximation was no longer accurate. However, we were pleased to find that the trajectory for the population size and mean phenotype for the analytical model agreed well with our finite-locus model when the viability selection was weaker so that more than $1 \%$ survived. Interestingly even then, the analytical approximation for the mean population size was nearly always slightly larger than that predicted by our finite-locus model and the mean phenotype reached the optimum much sooner (Fig. 3a).

We can suggest two explanations of why our finitelocus model predicts slower rates of evolution than the analytical model. First there is a synergistic effect of genetic and demographic stochasticity at the very small population sizes that follow a large shift in the optimum. After a large change in the optimal phenotype the population size becomes small. This reduces the rate of evolution and extends the time the population spends at small population sizes, where it is prone to extinction from demographic stochasticity and rapid loss of genetic variation from genetic drift. Bürger \& Lynch (1995) have previously noted this effect for evolution in response to continually moving optima.

Alternatively, the slower evolution in the finite-locus model may occur because it more realistically includes the 'Bulmer effect' (see Falconer \& MacKay, 1996) whereas the analytical model does not. The 'Bulmer effect' reduces the magnitude of the additive variance because of the generation of negative covariance between the effects of different trait loci under stabilizing selection (Bulmer, 1980). As we have shown, reduction in the initial level of additive genetic variance will slow down the rate of adaptation to the new optimum and reduce the magnitude of $\Delta \theta_{\mathrm{c}}$ that can be tolerated.

Gomulkiewicz \& Holt (1995) correctly observe that evolution causes almost no delay in the time critically low population sizes are first reached but incorrectly conclude that the main effect of evolution is to keep mildly maladapted populations from ever reaching such low levels. Our finite-locus model showed that a high initial heritability had no effect on the initial drop in population size one generation after the shift in the optimum. However, a high heritability greatly reduced the time the population spent at very small population sizes (Fig. 1). These differences between our conclusions and those of Gomulkiewicz \& Holt (1995) probably occur because we were able to monitor population sizes and extinctions directly rather than using their method of equating extinction risk to the period the 
population spent below an unknown critical population size, $\mathrm{N}_{\mathrm{c}}$. Plotting the transient dynamics of our finitelocus model shows that the probability of extinction is closely related to the mean minimum population size reached for a particular set of parameter values even though, for the parameter values used in the basic model, populations only stayed at those minimum population sizes for a few generations (e.g. Fig. 1). The dependence of extinction risk on the minimum population size reached was evident whether selection intensity was weak or strong.

Our results apply only when the assumptions of our finite-locus model are upheld. In particular we assume that genetic change occurs largely because of small changes in allele frequencies at a large but finite number of loci each having an equal but small effect on a single trait. We did not try to make the loci contribute unequally to the trait but believe this would be equivalent to having fewer loci and would therefore have little effect. We are only using a simple form of mutation but our results suggest that mutation is unlikely to be important on the short time scales that are important here. We also have only considered selection on a single trait even though selection on multiple, genetically correlated traits may be common in nature.

Our results suggest that the conservation of populations of species with low potential fecundities is inherently more difficult than those of species with high potential fecundities because they are more likely to go extinct after a discrete change in the environment. Of the genetic and demographic parameters that can be manipulated by conservation managers, our results show that maintaining a large population size and high levels of additive genetic variance are critical if the population is to adapt to discrete shifts in environmental conditions caused by a move to new habitat or exposure to new disease, competitors, or predators. Our model also suggests caution about maintaining genetic diversity by deliberately transplanting individuals among subpopulations (e.g. Nunney \& Campbell, 1993). If the local selective pressures are different in the different subpopulations then our results suggest that transplants are likely to imperil the subpopulations instead of rescuing them.

\section{Acknowledgements}

We thank B. Anholt, N. Barton, T. Crease, J.P. Gibson, B. Husband, D. Houle, A.S. Kondrashov, B.D. Roitberg, and C. Smith for reviewing various versions of this manuscript. Financial assistance was provided by N.S.E.R.C. (Canada) operating and equipment grants to E. G. Boulding.

\section{References}

BOAG, P. T. AND GRANT, P. R. 1981. Intense natural selection in a population of Darwin's ground finches (Geospiza) on Isla Daphne Major, Galapagos. Science, 214, 82-85.

BOULDING, E. G. 1990. Are the opposing selection pressures on exposed and protected shores sufficient to maintain genetic differentiation between gastropod populations with high intermigration rates? Hydrobiologia, 193, 41-52.

BOYCE, M. S. 1992. Population viability analysis. Ann. Rev. Ecol. Syst., 23, 481-506.

BUlmer, M. 1980. The Mathematical Theory of Quantitative Genetics. Clarendon Press, Oxford.

BÜRGER, R. AND LYNCH, M. 1995. Evolution and extinction in a changing environment. Evolution, 49, 151-163.

CHARLESWORTH, B. 1993. Directional selection and the evolution of sex and recombination. Genet. Res., 61, 205-224.

ENDler, J. A. 1986. Natural Selection in the Wild. Princeton University, Princeton, NJ.

FALCONER, D. S. AND MACKAY, T. C. 1996. Introduction to Quantitative Genetics, 4th edn. Longman, London.

GABRIEL, W., LYNCH, M. AND BÜRGER, R. 1993. Muller's ratchet and mutational meltdowns. Evolution, 47, 1744-1757.

GOMULKIEWICZ, R. AND HOLT, R. D. 1995. When does evolution by natural selection prevent extinction? Evolution, 49, 201-207.

GRANT, P. R. 1986. Evolution and Ecology of Darwin's Finches. Princeton University Press, Princeton, NJ.

KIRKPATRICK, M. AND BARTON, N. H. 1997. Evolution of a species' range. Am. Nat., 150, 1-23.

KONDRASHOV, A. S. AND YAMPOLSKY, L. Y. 1996. High genetic variability under the balance between symmetric mutation and fluctuating stabilising selection. Genet. Res., 68, 157-164.

LANDE, R. 1976. Natural selection and random genetic drift in phenotypic evolution. Evolution, 30, 314-334.

LANDE, R. 1988. Genetics and demography in biological conservation. Science, 241, 1455-1460.

LANDE, R. 1993. Risks of population extinction from demographic and environmental stochasticity and random catastrophes. Am. Nat., 142, 911-927.

LANDE, R. AND SHANNON, s. 1996. The role of genetic variation in adaptation and population persistence in a changing environment. Evolution, 50, 434-437.

LATTER, B. D. H. 1970. Selection in finite populations with multiple alleles. II. Centripetal selection, mutation, and isoallelic variation. Genetics, 66, 165-186.

LYNCH, M. 1996. A quantitative-genetic perspective on conservation issues. In: Avise, J. C. and Hamrick, J. L. (eds) Conservation Genetics: Case Histories from Nature, pp. 471-501. Chapman \& Hall, New York.

LYNCH, M. AND LANDE, R. 1993. Evolution and extinction in response to environmental change. In: Kareiva, P. M., Kingsolver, J. G. and Huey, R. B. (eds) Biotic Interactions and Global Change, pp. 234-250. Sinauer, Sunderland, MA.

LYNCH, M., GABRIEL, w. AND wood, A. M. 1991. Adaptive and demographic responses of plankton populations to environmental change. Limnol. Ocean., 36, 1301-1312. 
NUNNEY, L. AND CAMPBell, K. A. 1993. Assessing minimum population size: demography meets population genetics. Trends Ecol. Evol., 8, 234-239.

PEASE, C. M., LANDE, R. AND BULl, J. J. 1989. A model of population growth, dispersal, and evolution in a changing environment. Ecology, 70, 1657-1664.

SLATKIN, M. 1978. Spatial patterns in the distribution of polygenic characters. J. Theor. Biol., 70, 213-228.
UimARI, P., KenNedy, B. W. AND DEKKeRs, J. C. M. 1996. Power and parameter estimation of complex segregation analysis under a finite locus model. Génét. Sél. Évol., 28, 345-358.

YOUNG, S. S. Y. 1965. Computer simulation of directional selection in large populations I. The programme, the additive and the dominance models. Genetics, 53, 189-205. 